\title{
Circuit
}

Musiques contemporaines

\section{Introduction. Art + son $=$ art sonore ?}

\section{Annelies Fryberger}

Volume 30, numéro 1, 2020

Art + son $=$ art sonore $?$

URI : https://id.erudit.org/iderudit/1069079ar

DOI : https://doi.org/10.7202/1069079ar

Aller au sommaire du numéro

Éditeur(s)

Circuit, musiques contemporaines

ISSN

1183-1693 (imprimé)

1488-9692 (numérique)

Découvrir la revue

Citer ce document

Fryberger, A. (2020). Introduction. Art + son = art sonore ? Circuit, 30(1), 7-9. https://doi.org/10.7202/1069079ar

Ce document est protégé par la loi sur le droit d'auteur. L'utilisation des services d'Érudit (y compris la reproduction) est assujettie à sa politique d'utilisation que vous pouvez consulter en ligne.

https://apropos.erudit.org/fr/usagers/politique-dutilisation/
Cet article est diffusé et préservé par Érudit.

Érudit est un consortium interuniversitaire sans but lucratif composé de l’Université de Montréal, l'Université Laval et l'Université du Québec à Montréal. Il a pour mission la promotion et la valorisation de la recherche. https://www.erudit.org/fr/ 


\title{
Introduction. Art + son $=$ art sonore $?$
}

\author{
Annelies Fryberger
}

Tiens, qu'est-ce que c'est que ça? Un numéro de Circuit avec des textes venant d'historiens de l'art... Qu'est-ce qu'ils ont à dire sur la musique contemporaine? C'est de l'art sonore qu'ils parlent? Mais c'est quoi ça, au juste? Tout le monde en parle! D'ailleurs, j’ai vu une expo avec un corbeau mécanisé qui disait des choses étranges. C'est ça, de l'art sonore? Oui? Pas tout à fait? Bon...

Étonnant, peut-être, un numéro de Circuit dans lequel les musicologues sont minoritaires, où l'on voit le son abordé par des perspectives pas entièrement musicales. Il y est question du son... Il y est aussi question de l'art contemporain et de musées ${ }^{1}$. Le titre provocateur - «Art + son = art sonore? » - vient d'un colloque organisé à l'Ircam en $2018^{2}$, dans lequel des auteurs de ce numéro (Christophe Kihm et Lina Džuverović) ont pris la parole. Ce titre est censé nous laisser perplexes sur l'objet même qui est à l'étude: l'art sonore. Les contributions à ce numéro vont nous guider vers une compréhension plus globale de cette appellation et ses problématiques, et nous faire mieux comprendre ce qui se passe sur le plan institutionnel quand on présente un travail artistique pluridisciplinaire comme l'art sonore. Car c'est bien de pluri/ multi/transdisciplinarité qu'il s'agit ici, et de tous les problèmes qu'elle pose. Qui a le dernier mot sur une production artistique qui ne rentre pas dans les cases établies et compréhensibles pour le plus grand nombre, comme celles de l'art contemporain ou de la musique contemporaine? Puisqu'il s'agit ici du son, mais dans un contexte plutôt dédié au visuel - le musée (ou la galerie) -, est-ce aux musicologues de juger de la valeur de ces œuvres, ou plutôt aux historiens de l'art? Ceci implique une question corollaire: comment doit-on parler de ces œuvres? Quand on les analyse, comme le fait Karine P. Bouchard pour l'œuvre Telharmonium 2.o de Xavier Ménard et Camille
1. Je souhaite faire remarquer que Circuit a déjà abordé des thématiques proches de celle que nous traitons ici, notamment dans les numéros sur les "Musiques aux limites de l'image" (vol. 26, n ${ }^{3}$ ), "La musique des objets" (vol. 23, $n^{\circ}$ 1), et la "Musique in situ" (vol. 17, $\left.\mathrm{n}^{\circ} 3\right)$.

2. Voir: https://www.ircam.fr/agenda/ art-son-art-sonore/detail/ (consulté le 2 janvier 2020). 
3. "Klangkünstler, also jene Leute, die zwischen den artistischen Stühlen sitzen..." (Stefan Fricke [mai 2015], "Fluktuationen: Zur Klangkunst von José Antonio Orts", MusikTexte: Zeitschrift für neue Musik, nº 145, p. 15).

4. Andy Hamilton (2007), "Music and the Aural Arts", British Journal of Aesthetics, vol. 47, $\mathrm{n}^{0} 1$, p. 47.

5. David Toop (printemps 2005), "The art of noise", Tate Etc, $\mathrm{n}^{0} 3$, www.tate.org. uk/context-comment/articles/art-noise (consulté le 19 novembre 2019).

\author{
6. Gascia Ouzounian (février 2006), \\ "Embodied Sound: Aural Architectures \\ and the Body", Contemporary Music \\ Review, vol. 25, $\mathrm{n}^{0} 12$, p. 71. Terre \\ Thaemlitz, par exemple, cherche à \\ rendre visible l'espace institutionnel \\ quand il cache des boîtes émettant \\ un son de bip dans le MoMA en 1989 \\ (www.comatonse.com/thaemlitz/ \\ performances_more.html\#more \\ [consulté le 20 juillet 2018]).
}

St-Armand dans ce numéro, quel vocabulaire et quel bagage disciplinaire mobiliser?

On situe l'art sonore, typiquement, quelque part entre les arts plastiques et la musique contemporaine et/ou électronique, ou entre objet et performance, sans pouvoir lui donner une place définie; il est «entre deux chaises », pour reprendre l'expression du critique musical Stefan Fricke ${ }^{3}$. Cette identité floue implique une institutionnalisation complexe. Selon le point de vue adopté, on peut dire que l'art sonore fait entrer le sonore dans les arts plastiques ou, au contraire, qu'il fait entrer le visuel dans la musique. Son institutionnalisation est donc à double sens. Les festivals de musique contemporaine font de plus en plus la part belle aux installations sonores, et les musées et les galeries suivent le même mouvement - pourtant, les artistes qui réussissent à intégrer les deux circuits sont rares. Le son lui-même peut jouer des rôles différents. Ainsi, certains artistes sonores cherchent à libérer le son de l'hégémonie de la musique ${ }^{4}$, tandis que d'autres incluent l'art sonore dans leur pratique de composition (musicale). Ou encore, le son peut être représenté de manière muette, tandis qu'une image peut être musicale, comme on le voit dans les œuvres - réalisées spécialement pour ce numéro - de l'artiste hautement polyvalent L. L. de Mars, surtout connu pour ses bandes dessinées.

Du côté des arts plastiques (d'ailleurs toujours désignés comme visual arts en langue anglaise), le travail de Marcel Duchamp est souvent cité comme instigateur d'une ouverture envers l'élément sonore dans l'espace muséal'5. Plus tard, avec le travail de Joseph Beuys et le mouvement Fluxus, on a commencé à voir les premières entrées du bruit dans les musées et les galeries. Plus récemment, surtout à partir des années 2000 , une vague de grandes expositions sur les arts sonores - avec l'utilisation spécifique de ce terme - a déferlé; il s'agit d'un lent processus d'institutionnalisation de cette forme artistique dans le cadre du musée. Quels sont alors les tensions produites par ce processus, ou les changements dans la conception du lieu induits par l'inclusion d'éléments sonores? Lart sonore peut montrer la façon dont l'espace est lui-même construit socialement et politiquement ${ }^{6}$. Lina Džuverović étudie la manière dont le son, avec son potentiel à éviter l'objet et ses propriétés collaboratives intrinsèques, sait ouvrir de nouvelles avenues de solidarité et de collectivité, ou un lieu de résistance contre le milieu de l'art contemporain. Christophe Kihm propose quant à lui d'explorer les premières entrées significatives du son en contexte muséal en revenant sur deux expositions en miroir, Für Augen und Ohren et Écouter par les yeux, qui s'intéressaient respectivement aux rapports de la musique et des arts plastiques au $\mathrm{xx}^{\mathrm{e}}$ siècle et au «matériau son» dans les arts. Le flou entourant la notion d'art sonore 
est au cœur de son essai, et il nous invite à revoir ce problème de définition en nous ouvrant à l'écoute et à l'effet décentrant qu'elle peut avoir.

Du côté de la musique contemporaine, on retrace les origines des arts sonores dans les forces modernistes du début du xx $x^{\mathrm{e}}$ siècle, avec les futuristes italiens souvent nommés comme précurseurs de ce type d'expression artistique. Le modernisme en général - et les futuristes en particulier - ont connu une suite hautement problématique sur le plan politique, avec le tournant fasciste des futuristes et leur rejet explicite du féminisme ${ }^{7}$. Lart sonore, dans sa nature même, questionne les tenants du modernisme, en déstabilisant l'auctorialité par le biais des collaborations (entre artistes de disciplines différentes et aussi avec le public) ou en revoyant la notion de l'œuvre. L'art sonore s'avérera-t-il un havre pour ceux et celles qui souhaitent se débarrasser de l'héritage moderniste problématique? En fournissant la possibilité d'interroger les catégories historiques de l'œuvre et de l'auteur, l'art sonore permettra-t-il aux minorités de trouver plus facilement leur place dans ce milieu - voire de nous faire sortir de l'anthropocentrisme ${ }^{8}$ ? Surtout si on le compare à la musique contemporaine ou aux arts plastiques, qui s'inscrivent tous deux dans une longue tradition de domination masculine et européenne dont il semble difficile de se débarrasser?.

Le fait de voir l'art sonore comme un genre à part ne va pas de soi. C'est une forme d'expression qui est de nos jours intimement liée aux institutions qui l'accueillent, qu'elles soient musicales ou artistiques. Ce fait a un impact sur la manière dont les artistes conçoivent leur propre pratique, ce que Clément Canonne et moi explorons dans ce numéro. Ana Dall'Ara-Majek montre, pour sa part, comment les artistes sonores de différentes générations naviguent dans ce paysage institutionnel complexe. D'un point de vue sociologique, l'art sonore souffre d'une difficulté de catégorisation ${ }^{10}$, ce qui rend son évaluation complexe, notamment dans les organes de soutien pour la musique ou l'art contemporains ${ }^{11}$. Le désir de doter cette forme artistique d'un nom spécifique vient peut-être d'une "angoisse de genre ${ }^{12}$ », dans le sens où elle a besoin d'un nom pour ne pas être ignorée ou dissolue dans les genres préexistants. Toutefois, il se peut que ce genre se définisse davantage par les moyens techniques de sa diffusion que par ses caractéristiques conceptuelles ou esthétiques.

Qu'il soit défini ou pas, ce genre se fait entendre et fascine. En avant, les oreilles et les yeux ouverts!

Bonne lecture!

New York, novembre 2019
7. Jahan Ramazani et al. (2003), "Introduction", in Jahan Ramazani et al. (dir.), The Norton Anthology of Modern and Contemporary Poetry, New York, W.W. Norton \& Company, p. Ix.

8. Voir l'article de Christophe Kihm dans ce numéro.

9. Voir par exemple Lauren Redhead (2015), " "New Music" as Patriarchal Category", in Catherine Haworth et Lisa Colton (dir.), Gender, Age and Musical Creativity, Surry, Ashgate, p. 171-183.

10. Ezra W. Zuckerman (1999), "The Categorical Imperative: Securities Analysts and the Illegitimacy Discount ", American Journal of Sociology, vol. 104, $\mathrm{n}^{\circ}$ 5, p. 1398-1438.

11. Annelies Fryberger (2016), "L'évaluation par les pairs en musique contemporaine en France et aux États-Unis", Circuit, musiques contemporaines, vol. $26, n^{\circ} 2$, p. 15-27. 12. Andy Hamilton, op. cit., p. 63. 